\section{Novel imaging technique could lead to early diagnosis of lung cancer}

\begin{tabular}{|c|c|}
\hline $\begin{array}{l}\text { Non-small cell lung cancer is } \\
\text { one of the most widespread } \\
\text { forms of the disease. As with } \\
\text { many other cancers, the best } \\
\text { chance of survival comes with } \\
\text { early diagnosis; unfortunately, } \\
\text { at present this often doesn't } \\
\text { happen. In research that has } \\
\text { the potential to improve the } \\
\text { prognosis of lung cancer } \\
\text { patients, Dr Claudio Scafoglio } \\
\text { and colleagues at the } \\
\text { University of California, Los } \\
\text { Angeles, has identified a new } \\
\text { diagnostic technique that could } \\
\text { efficiently identify the earliest } \\
\text { stages of lung cancer. }\end{array}$ & $\begin{array}{l}\text { ancer comes in many forms. } \\
\text { Worldwide, lung cancer is the } \\
\text { most prevalent form of the } \\
\text { disease, followed by breast, bowel and } \\
\text { prostate cancer. There are two types of } \\
\text { lung cancer: small cell lung cancer and } \\
\text { non-small cell lung cancer (NSCLC). } \\
\text { Of the two, NSCLC is by far the most } \\
\text { common, accounting for around } 80 \text { to } \\
85 \% \text { of lung cancer cases. } \\
\text { Despite huge advances in cancer } \\
\text { treatment, one factor still gives the } \\
\text { best chance of surviving NSCLC: early } \\
\text { diagnosis. While more than half of } \\
\text { NSCLC patients with localised disease } \\
\text { (i.e. where the cancer has not yet spread } \\
\text { from the lungs) will survive for at least } \\
\text { five years, only } 4.5 \% \text { of patients with } \\
\text { the metastatic form of the disease can } \\
\text { expect to live that long. Unfortunately, at }\end{array}$ \\
\hline
\end{tabular}

present relatively few newly-diagnosed cases, the cancer has already spread or metastasised. To improve survival rates, it is crucial that new routes to early diagnosis are explored.

\section{SEARCHING FOR THE EARLIEST} SIGNS OF CANCER

To diagnose cancer at an early stage, doctors sometimes look for signs of premalignancy. Premalignant cells are those which are likely to become cancerous, but have not yet done so. In some types of lung cancer, signs of premalignancy can be detected by computed tomography (CT) scans. insufficient, as it can be afficult to tell whether a particular lesion is cancerous or not There are two main types of NSCLC. lung adenocarcinoma and squamous cell carcinoma. Lung adenocarcinoma is more challenging to diagnose as it typically develops in the furthest reaches of the lungs, the bronchioles and alveoli. There are several types of premalignant lesion that are known to predict the emergence of lung adenocarcinoma; unfortunately, detection

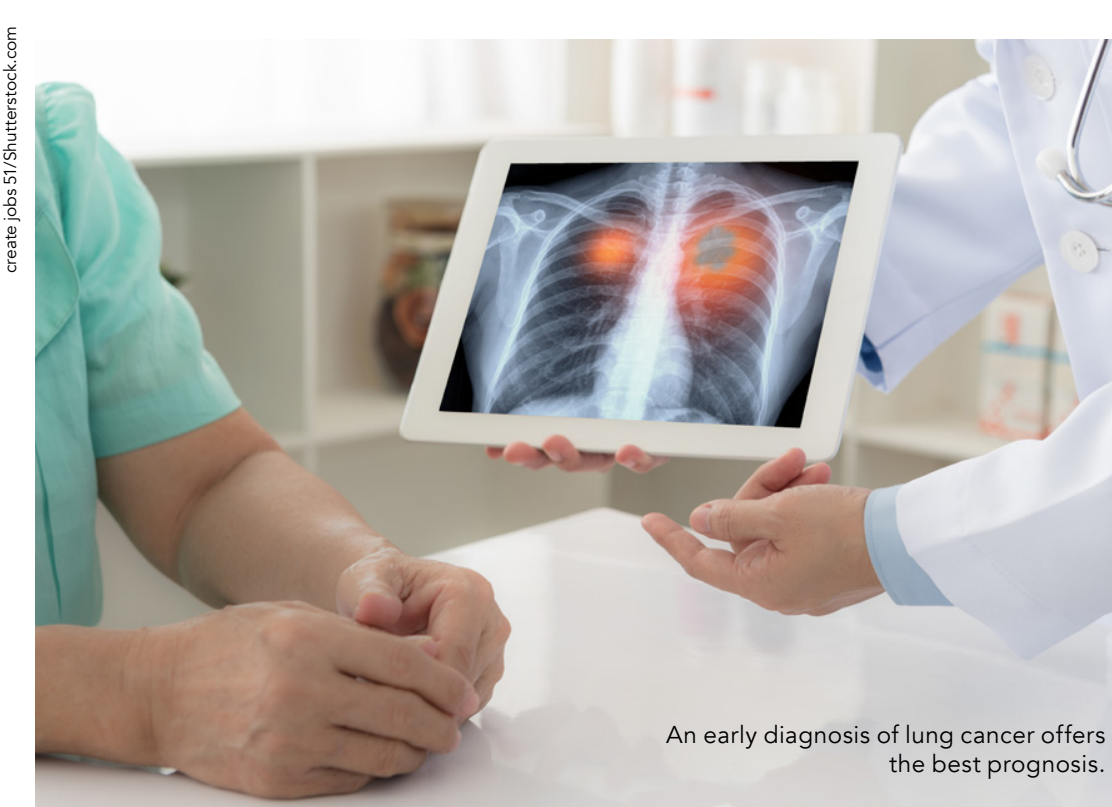

SGLT2 appears to be most active in the first stages of the disease, during premalignancy and the beginnings of tumour development.

of the disease. A particular group of transporter molecules, called GLUTs, are responsible for carrying glucose into cancer cells. One way to search for the presence of cancer is to inject a patient with a small amount of a radioactive glucose substitute, or tracer. GLUTs carry the radioactive molecules into cancer cells, which can then be detected with a type of radioactivitydetecting scan called positron emission tomography (PET). RET is useful for cancer stagng, or determining how around the body. A negative PET scan indicates that the cancer rem confined to the original tuno not yet metastasised.

While traditional PET scanning is helpful for more advanced cancers, it is not useful for identifying early-stage lung adenocarcinoma. Dr Claudio Scafoglio and his team at the University of California believe that this is because this form of the disease uses a differen system to transport glucose into cancerous cells: sodium-dependent glucose transporters, or SGLTs. These transporters, unlike GLUTs, cannot carry the radioactive glucose substi

\section{A NEW WAY TO}

DETECT MALIGNANCY

Scafoglio and his team investigated whether SGLTs are a sign of premalignancy and the early this, the researchers used a different tris, the researchers used a different transported by SGLTs but not by GLUTs.

The team looked at one particular SGLT, called SGLT2. Dr Scafoglio and his colleagues had previously

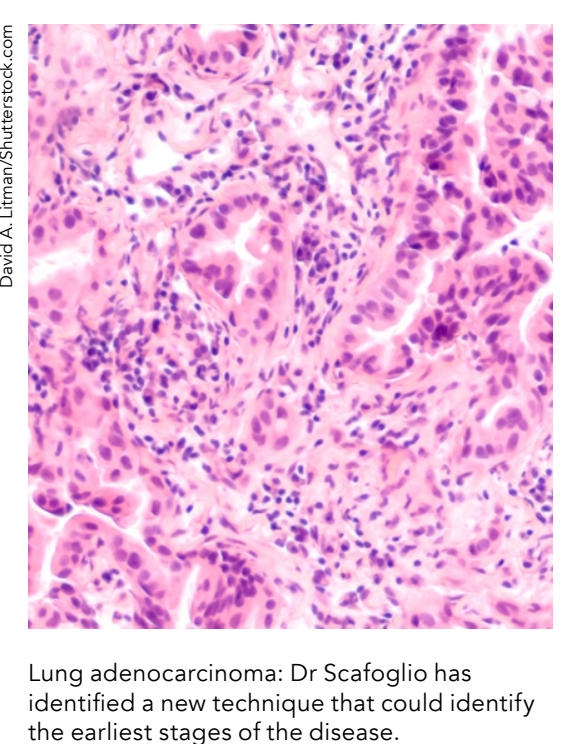

dentified this transporter in differen and prostate tumours. In this study they used a technique called to search lung adenocarcinoma samples. Immunohistochemistry is a method for selectively staining, or dying, tissue samples. The protein of interest - in this case, SGLT2 - is marked with a stain which is visible when the tissue sample is viewed with a microscope. This allows researchers to observe the abundance and distribution of the protein.

In total, the team examined 58 lung pactecarcinoma samples, taken from patients at disferent stages of the disease. They also used geneticallythe SGLT2 protein. Finally, the

researchers developed patient-derived xenografts (PDXs). A PDX is created when tumour tissue is taken from a patient and implanted into mice for research purposes. This technique allows drugs to be tested in mice before they are given to patients.

The in-depth methods allowed the researchers to demonstrate that

SGLT2 does play an important role in lung adenocarcinoma. Interestingly, SGLT2 appears to be most active in the first stages of the disease, during premalignancy and the beginnings cancer cells seem to have only SGIT whereas cells in more advanced thours have both SGIT2 and GIUTS.

This means that the ways in which

cancer cells take in glucose change

as the disease progresses. Why this happens is unclear. However, Dr

Scafoglio thinks that the reason may lie in the varying levels of oxygen available to cancer cells at differen stages of the disease. Early cancer cells have a good oxygen supply, while those at a more advanced stage must survive in a low-oxygen environment It may be that GLUTs are better able to function in these conditions. Dr Scafoglio and his team recently received a grant from he US National Cancer Institute, which will allow them lung cancer progression. 
FDG
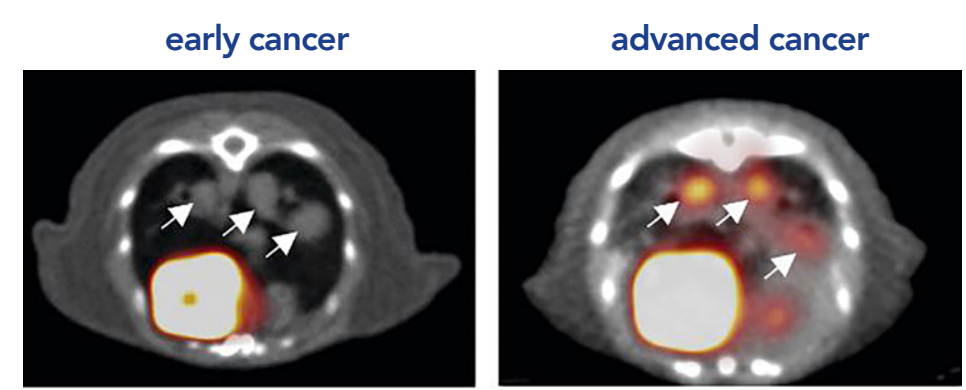

Me4FDG
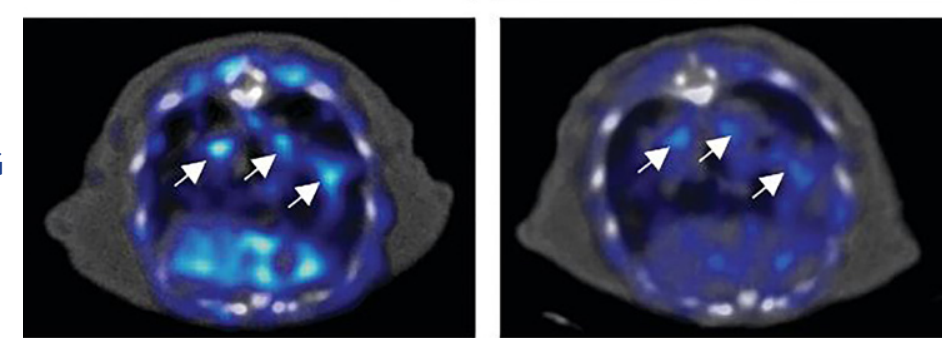

A new PET tracer can visualise tumours that are negative with traditional PET. The two different
ETT tracers, FDG (the traditional one, upper panels) and Me $F F D G$ (the new experimental one lower pans at different times, when the tumours are small (left panels) and when they are more advanced
(ight panels). The tumours, highlighted by the white arrows, show untake only yof the new traccer (right panels). The tumours, , highlighted by the white arrows, show uptake only of the new tracer
(Me $4 F D G$, in blue) when they are at an early stage, and both the new and the traditional tracer
(FDG,

BETTER DIAGNOSIS COULD LEAD TO IMPROVED SURVIVAL RATES The research led by Dr Scafoglio

most of which turn out to be benign. reliable diagnostic methods for lung adenocarcinoma. One current problem with CT scanning is that the technique identifies large numbers of lung nodules, easily distinguished from cancerous cells, scans to check that the nodular $\mathrm{CT}$ becoming malignant; understandably, this uncertainty creates a great deal of
anxiety and stress for patients. By using

Drugs which block the development of SGLT transporters can effectively hinder disease progression, by limiting the glucose supply of cancer cells.

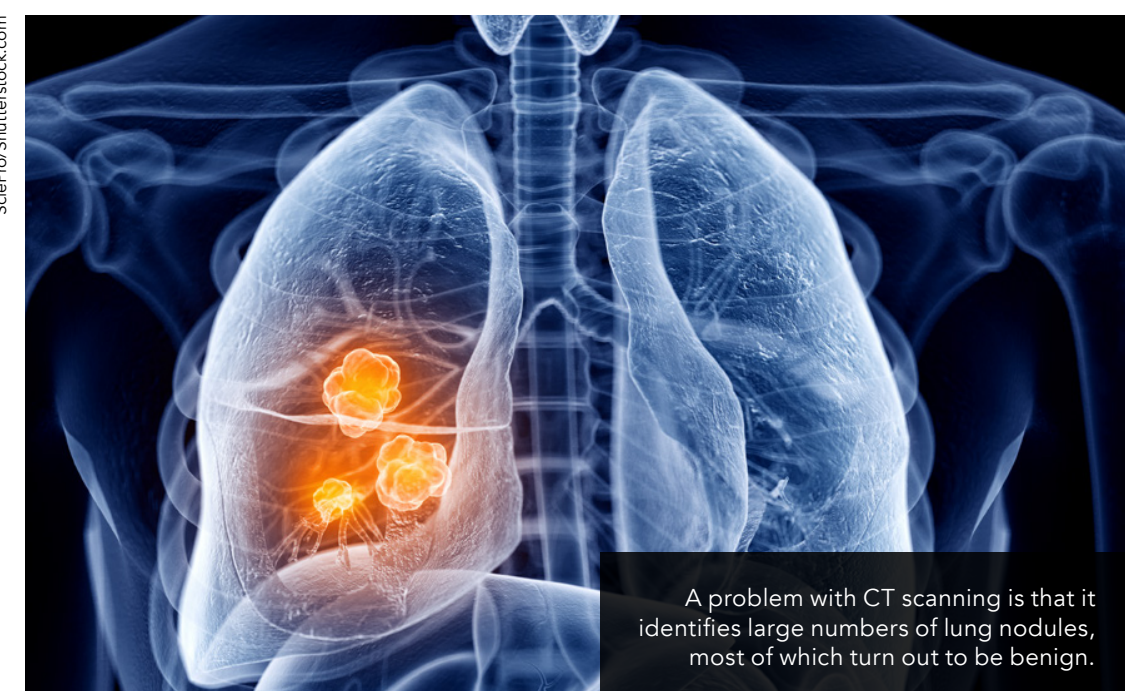

the alternative tracer that is taken up ersty-stage tremalignant lesions and identified This will allow patients with lung adenocarcinoma to receive an early diagnosis, and consequently lead to a better chance of survival. At the same me, patients with benign lung lesions will be spared unnecessary treatment

The team also discovered that drugs which block the development of SGLT ransporters can effectively hinder disease progression, by limiting the glucose supply of cancer cells. These drugs, which can help to regulate blood sugar levels, are already available for treating diabetes. In this study, the researchers found that these drugs can specifically target premalignant lesions in the lungs; given early, he treatment could improve even have the potential to prevent premalignant cells from becoming cancerous at all. They could be particularly useful in patients for whom surgical removal of the lesion would be risky.

Finally, the team propose combining their two key discoveries. PET scanning with the alternative tracer used in this study could be used both before and after a patient receives SGLT-blocking drugs, to monitor changes in the progression of the disease. This has the potential to be a diagnostic tool which offers a degree of precision that is not currently available. With more detalled information of the state of a patient's cancer, doctors will be better equipped to decide on the most

Following the promising results of this study, the team's next goal is to of this clinical trials to assess the use of the new tracer in patients. This is crucial if the new diagnostic method outlined here is to become a reality for cancer patients and their doctors. Given the global prevalence of NSCLC, advances in diagnosis and treatment, such as those pioneered by Dr Scafoglio and his colleagues, have real potential to reduce the number of people experiencing this disease. As with many other cancers, the key to a person's chances of survival from NSCLC is an early diagnosis - and the research of $D$ Scafoglio and his colleague

\section{ore Behind the Research}

. Dr Claudio Scafoglio

E: cscafoglio@mednet.ucla.edu T: +1 310-825-9577 W: www.scafogliolab.org W: https://www.researchgate.net/profile/Claudio_Scafoglio

\section{Research Objectives}

Claudio Scafoglio's research is focused on

understanding the utilisation of glucose by cancer cells.

\section{Detail}

Claudio Scafoglio

Avenue, BH-127 CHS UCLA David Geffen school of Medicine Los Angeles, CA 90095 USA

Bio

Claudio Scafoglio completed his Medical Degree and PhD Degree at the University of Napoli, Italy. He received postdoctoral training at UCSD on gen regulation in cancer and at UCLA in cancer molecular imaging. His laboratory at UCLA is focused on early lung cancer imaging and treatment.

Funding

American Cancer Society

Tobacco Related Disease Research Program

National Center for Advancing Translational Sciences

\section{Collaborators}

- Jorge Barrio

- Steven Dubine

- Dane Yanagawa

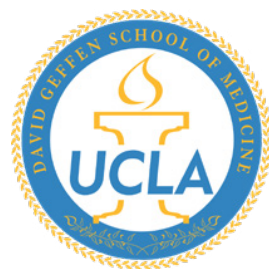

\section{References}

Scafoglio, CR, et al. 2018. Sodium-glucose transporter 2 is a diagnostic and therapeutic target for early-stage lung cancer. Sci Trans/ Med 10. (467):eaat5933. doi: $10.1126 /$ scitranslmed.aat5933.

\section{Personal Response}

How big an impact could these discoveries have on the prognosis of patients with NSCLC?

II The lung screening by CT has been estimated to

reduce lung cancer mortally by $20 \%$. By helping the improve the survival of NSCLC patients. The most exciting aspect of our research is the coupling of the diagnostic probe with a therapy specifically targeting tha gluce been unsuccessful; our research could introduce a new strategy of targeted chemoprevention: we could identify individuals with early-stage lesions that would be likely to benefit from chemoprevention with glucose
transporter inhibitors.

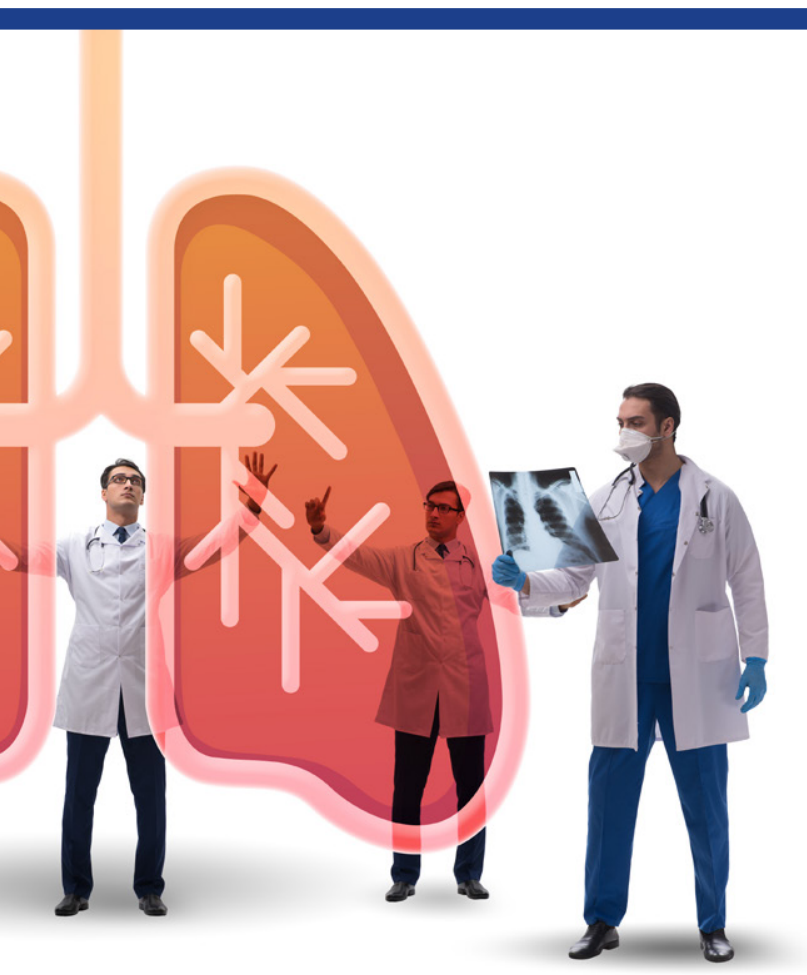

